\title{
Modélisation de la distribution potentielle de Cedrus atlantica Manetti au Maroc et impacts du changement climatique
}

\author{
Said MOUKRIM ${ }^{1,2}$ \\ Said LAHSSINI ${ }^{3}$ \\ Nabil RIFAI ${ }^{2}$ \\ Kamal MenzoU ${ }^{2}$ \\ Hicham MHARZI-ALAOUI ${ }^{2}$ \\ Adnane LABBACI ${ }^{4}$ \\ Mouhssine RHAZI ${ }^{5}$ \\ Imane WAHBY ${ }^{1}$ \\ Mohammed EL MADIHI ${ }^{1}$ \\ Laïla RHAz $^{1}$
}

${ }^{1}$ Université Mohammed V de Rabat

Faculté des Sciences

Centre de recherche BIOBIO

Avenue Ibn-Battouta, BP. 1014 RP.

Rabat, Maroc

${ }^{2}$ Institut agronomique et vétérinaire Hassan II

Département des Eaux et Forêts

3, Rue Haroun Errachid

Rabat, Maroc

${ }^{3}$ École nationale forestière d'ingénieurs BP 511, Tabriquet

11000 Salé, Maroc

${ }^{4}$ Université Ibn Zohr

BP 32/S, Riad Salam

80000 Agadir, Maroc

${ }^{5}$ Université Moulay Ismail

Faculté de sciences et techniques

d'Errachidia

BP 509, Boutalamine

52000 Errachidia, Maroc

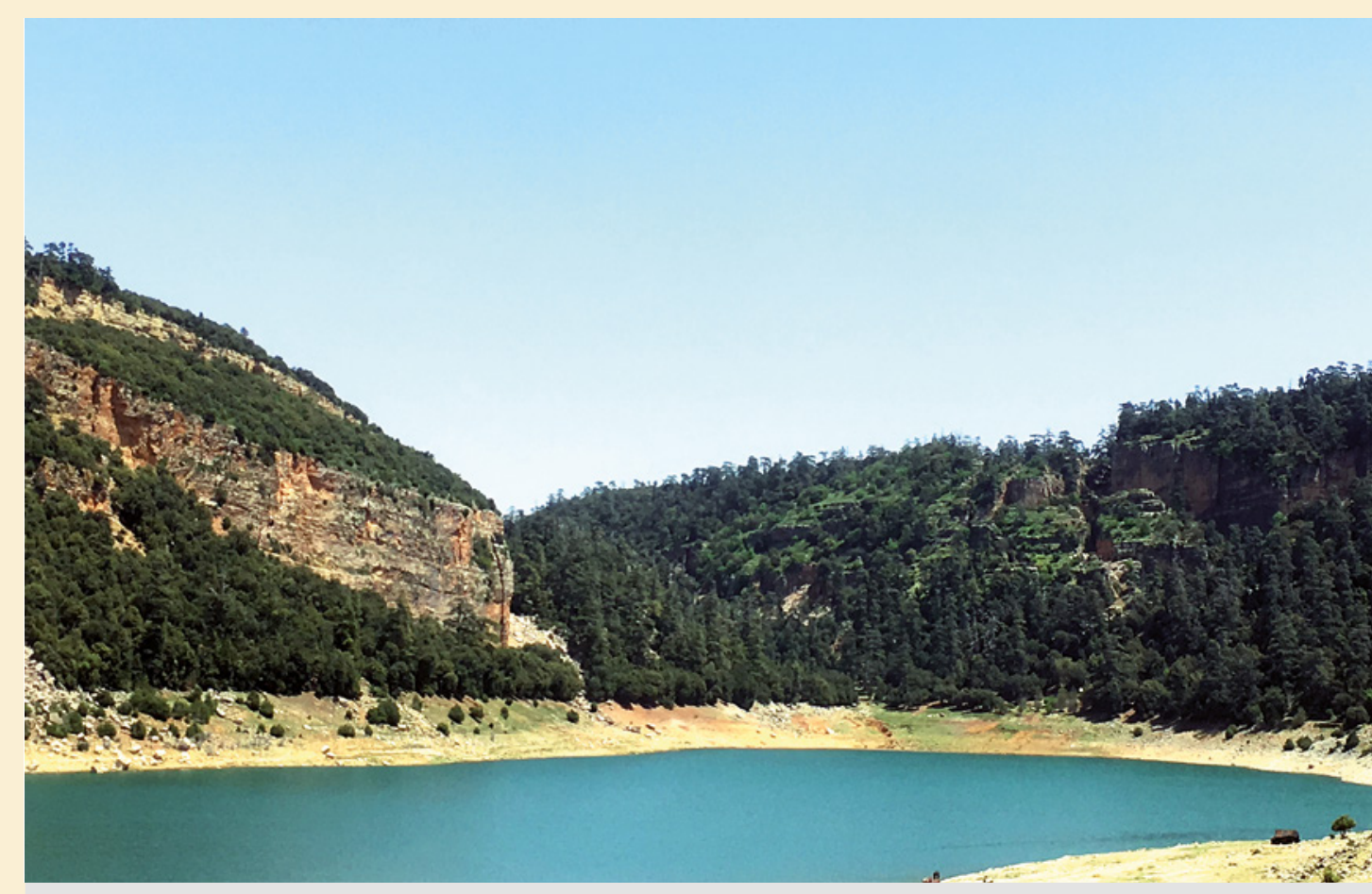

Auteur correspondant /

Corresponding author:

Said Moukrim -

maildemoukrim@gmail.com

Photo 1.

En pleine cédraie du Moyen Atlas, le site Aguelmam Azegza s'étend sur une superficie d'environ $40 \mathrm{ha}$, au milieu duquel se trouve le lac naturel Azegza (signifiant bleu en langue amazigh). Berceau d'une grande diversité faunistique et floristique, ce site reste vulnérable aux changements climatiques.

Photo S. Lahssini.

Doi : 10.19182/bft2020.344.a31888 - Droit d'auteur @ 2020, Bois et Forêts des Tropiques @ Cirad - Date de soumission : 20 septembre 2019 ; date d'acceptation : 28 janvier 2020 ; date de publication : $1^{\text {er }}$ mai 2020.

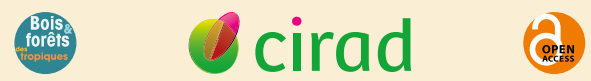

Licence Creative Commons :

Attribution 4.0 International (CC BY 4.0).
Citer l'article / To cite the article

Moukrim S., Lahssini S., Rifai N., Menzou K., Mharzi-Alaoui H., Labbaci A., Rhazi M., Wahby I., El Madihi M., Rhazi L., 2020. Modélisation de la distribution potentielle de Cedrus atlantica Manetti au Maroc et impacts du changement climatique. Bois et Forêts des Tropiques, 344 : 3-16. Doi: https://doi.org/10.19182/bft2020.344.a31888 


\section{RÉSUMÉ}

\author{
Modélisation de la distribution \\ potentielle de Cedrus atlantica Manetti \\ au Maroc et impacts du changement \\ climatique
}

Le changement climatique constitue un grand défi à relever par les politiques publiques et les gestionnaires des espaces naturels. La compréhension de ses impacts sur les écosystèmes forestiers constitue un préalable nécessaire à toute réflexion visant leur conservation et le maintien des services écosystémiques qu'ils offrent. Cette étude s'est intéressée à la modélisation de la distribution potentielle de Cedrus atlantica Manetti, à l'identification des variables environnementales conditionnant sa distribution, et à la définition de l'étendue géographique des aires qui sont favorables à sa présence sous les conditions climatiques passées, actuelles et futures. Ce travail a été réalisé en se basant sur le principe d'entropie maximale. La cartographie des aires potentielles révèle la sensibilité du cèdre aux changements climatiques passés et suggère une grande étendue (quadruple de la surface d'occurrence actuelle de l'espèce) des zones bioclimatiquement adaptées au développement de cette espèce sous les conditions climatiques actuelles. Cependant, les distributions potentielles futures semblent être dépendantes de certains sites spécifiques et risquent d'être affectées négativement par le climat futur. Ces résultats mettent en évidence l'ampleur des régressions projetées des superficies du cèdre dans le futur, ce qui pourrait impacter considérablement les fonctions et services de cet écosystème et augmenter les risques d'extinction locale. Ils constituent par ailleurs un bon outil de prise de décision pour les gestionnaires du territoire quant à la conservation de ce précieux écosystème forestier, afin d'éviter toute extinction de ce foyer de biodiversité marocaine.

Mots-clés : Cedrus atlantica Manetti, cèdre de l'Atlas, modélisation de la distribution d'espèces, MaxEnt, changement climatique, conservation, Maroc.

\section{Modelling the potential distribution of Cedrus atlantica Manetti in Morocco and impacts of climate change}

Climate change is a major challenge for public policies and the management of natural areas. Understanding its impacts on forest ecosystems is essential to undertake any policy-making for their conservation and preservation of their ecosystem services. This study focused on modelling the potential distribution of Cedrus atlantica Manetti, by identifying the environmental variables governing its distribution and defining the geographical extent of suitable areas to its presence in past, present and future climatic conditions. The study was based on the Maximum Entropy Principle. Mapping of the potential areas of distribution showed the sensitivity of this cedar species to past climate changes and suggests that bioclimatically suitable zones for the development of this species under current climatic conditions cover a large area (four times the current area of occurrence of the species). However, the potential future distribution of the species seems to be dependent on certain specific sites and is liable to be negatively affected by future climatic conditions. Our results show the scale of the projected shrinking of areas suitable for this cedar species in the future, which could have a considerable impact on the functions and services provided by this ecosystem and worsen the risks of local extinction. They also make up a useful decision-support tool for the management of this valuable forest ecosystem to ensure its conservation and avoid the risk of extinction of this locus of biodiversity in Morocco.

Keywords: Cedrus atlantica Manetti, Atlas Cedar, modelling species distribution, MaxEnt, climate change, conservation, Morocco.

\section{Modelización de la distribución potencial del Cedrus atlantica Manetti en Marruecos e impacto del cambio climático}

El cambio climático constituye un gran reto a asumir por las políticas públicas y los gestores de espacios naturales. La comprensión de su impacto en los ecosistemas forestales constituye una condición previa necesaria para cualquier reflexión que apunte hacia la conservación y el mantenimiento de los servicios ecosistémicos que ofrecen. Este estudio se interesa por la modelización de la distribución potencial del Cedrus atlantica Manetti, la identificación de las variables medioambientales que condicionan su distribución y la definición de la extensión geográfica de las zonas que son adecuadas a su presencia en las condiciones climáticas pasadas, actuales y futuras. Este trabajo se basa en el principio de entropía máxima. La cartografía de las zonas potenciales revela la sensibilidad del cedro ante los cambios climáticos pasados y sugiere una gran extensión (cuádruple de la superficie de ocurrencia actual de la especie) de zonas bioclimáticamente adaptadas al desarrollo de esta especie en las condiciones climáticas actuales. Sin embargo, las distribuciones potenciales futuras parecen depender de ciertos lugares específicos y corren el riesgo de que les afecte negativamente el clima futuro. Estos resultados ponen en relieve la amplitud de las regresiones de las superficies de cedro proyectadas al futuro, cosa que podría impactar considerablemente las funciones y servicios de este ecosistema y aumentar el riesgo de extinción local. Constituyen, asimismo, una buena herramienta de toma de decisión para los gestores del territorio en cuanto a la conservación de este precioso ecosistema forestal, y para evitar el riesgo de extinción de este foco de biodiversidad marroquí.

Palabras clave: Cedrus atlantica Manetti, cedro del Atlas, modelización de la distribución de especies, MaxEnt, cambio climático, conservación, Marruecos. 


\section{Introduction}

Le changement climatique en cours, principalement d'origine anthropique, devient de plus en plus une préoccupation majeure affectant de nombreux aspects de la société humaine et des composants de la biosphère à l'échelle mondiale (MEA, 2005 ; IPCC, 2013, 2018 ; Hallegatte et al., 2015). La hausse des température et l'altération des régimes pluviométriques observés actuellement devraient se poursuivre pendant le siècle en cours (IPCC, 2018).

Ces changements climatiques affectent déjà de nombreux aspects du monde vivant et sont reconnus comme l'une des principales menaces vis-à-vis de la survie des espèces et de l'intégrité des écosystèmes (MEA, 2005 ; Hallegatte et al., 2015 ; IPCC, 2018). Ces changements sont d'ores et déjà visibles à la fois sur la physiologie, la phénologie et sur l'organisation écologique des espèces, entraînant ainsi des changements dans leur composition et dans leurs aires de distribution (Hughes, 2000 ; McCarty, 2001 ; IPCC, 2018).

Pour le Maroc, les projections climatiques annoncent des tendances à la hausse des températures et à la réduction du volume pluviométrique et du nombre de journées pluvieuses (IPCC, 2013 ; MDCE, 2016). Ces changements climatiques risquent d'entraîner des altérations (réductions ou expansions) des aires de distribution de certaines espèces forestières (MDCE, 2016 ; Gbètoho et al., 2017 ; Moukrim et al., 2019b).

La gestion durable des écosystèmes forestiers, dans le contexte du changement climatique (MDCE, 2016), implique la connaissance des aires de distribution potentielles passées, actuelles et futures, mais aussi l'identification des facteurs qui la conditionnent et la connaissance des changements attendus susceptibles de les affecter (Pearson et Dawson, 2003 ; Thuiller et al., 2008). De telles informations font défaut dans le contexte marocain, ce qui constitue un défi fondamental qui complique la tâche pour les scientifiques, les conservateurs et les gestionnaires des ressources forestières, lesquels doivent souvent œuvrer avec des données limitées pour étudier, restaurer et gérer durablement le patrimoine naturel que constituent ces écosystèmes.

La cédraie est un bon exemple de cette problématique. Cedrus atlan- tica Manetti est une essence forestière représentative des écosystèmes forestiers remarquables d'Afrique du Nord (Myers et al., 2000 ; Fennane et Ibn Tattou, 2012) qui assure des fonctions et services variés (protection, production, régulation, culturels, esthétiques...) (M’hirit et Benzyane, 2006). L'importance de cette essence a justifié la reconnaissance de cet écosystème en tant que patrimoine mondial en 2016, avec un statut spécial de réserve de biosphère. Malgré cela, cet écosystème reste soumis à diverses pressions anthropiques (surpâturage, ébranchage, délits forestiers et prélèvements illégaux de bois) et climatiques conduisant à son évolution régressive (M’hirit et Benzyane, 2006 ; Rhanem, 2011).

L'objectif de la présente étude est de modéliser la distribution potentielle de cette espèce sous les conditions climatiques passées (Holocène moyen : 6000 ans avant le présent), actuelles et futures (pour 2050 et 2070), et d'identifier les variables environnementales affectant sa répartition géographique. Ce travail vise aussi l'évaluation de l'impact du changement climatique sur cette distribution afin d'appuyer les processus de prise de décision actuels et futurs, en vue de mieux hiérarchiser les efforts de gestion à court et à moyen terme de cette espèce menacée.

Ce travail est basé sur la modélisation de la distribution des espèces (SDM, Species Distribution Modeling), qui représente une approche pertinente de prédiction en écologie de la conservation (Franklin et Miller, 2010 ; Guisan et al., 2017).

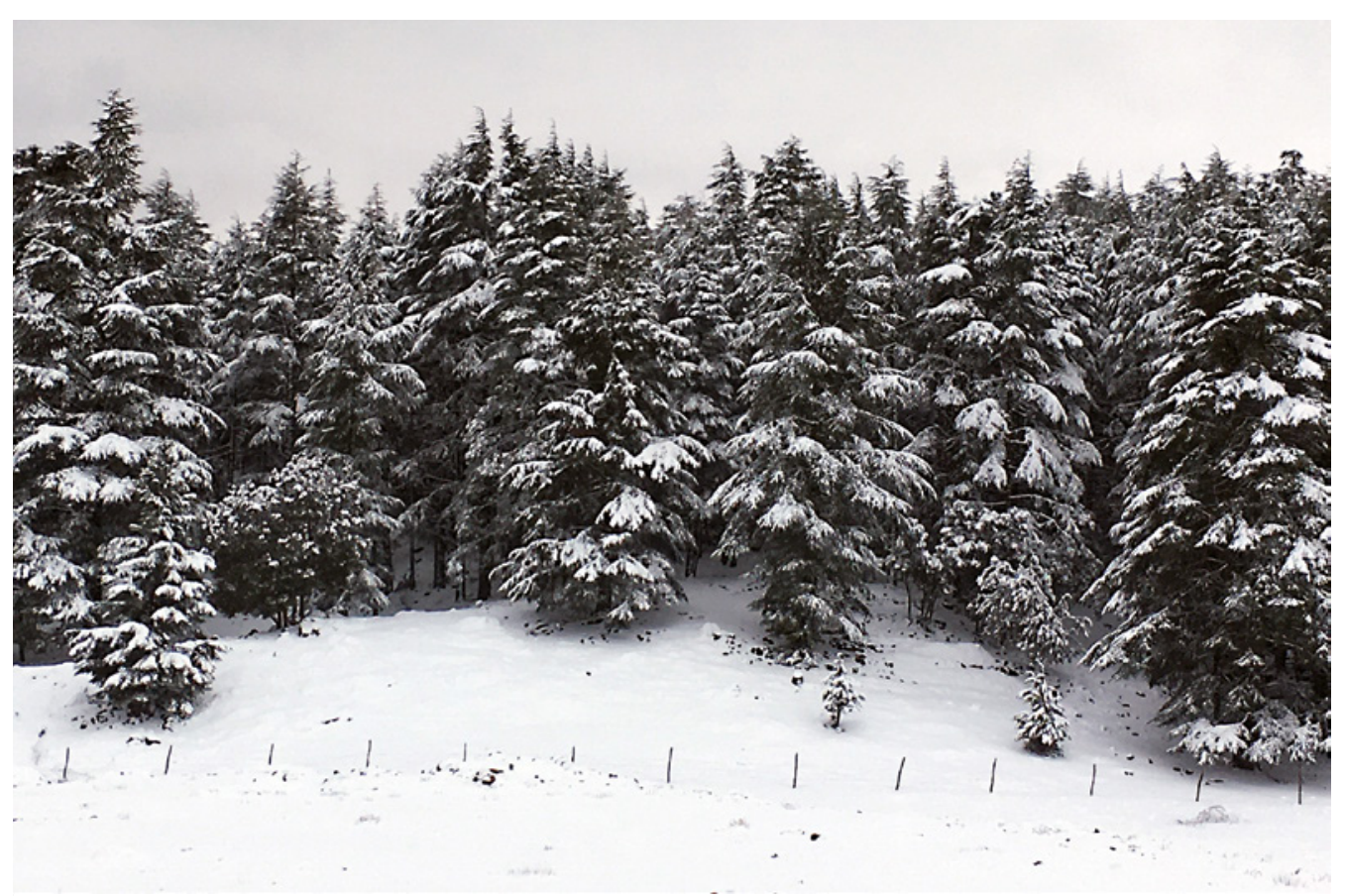

Photo 2.

Périmètre de régénération naturelle du cèdre. La clôture est installée pour bloquer l'intrusion des troupeaux des populations riveraines. Photo S. Lahssini. 


\section{Matériels et méthodes}

\section{Zone d'étude et écologie de l'espèce modèle}

La zone d'étude retenue concerne le territoire du Royaume du Maroc. Ce pays est situé à l'extrême nord-ouest de l'Afrique, entre $21^{\circ}$ et $36^{\circ}$ de latitude Nord et entre $1^{\circ}$ et $17^{\circ}$ de longitude Ouest. II jouit d'une position géographique à la rencontre de trois grands ensembles distincts, la Méditerranée au nord, l'océan Atlantique à l'ouest et le front désertique du Sahara au sud-est.

L'espèce étudiée dans le cadre de ce travail est le cèdre de l'Atlas. Cedrus atlantica Manetti est une espèce endémique des montagnes d'Afrique du Nord (photos 1 à 4). La répartition de son aire naturelle est discontinue, en sept blocs, et liée essentiellement à l'orographie du Maghreb (Boudy, 1950). Les trois premiers blocs se situent dans l'Atlas algérien sur environ 33000 ha (Rabhi et al., 2018). Les quatre autres blocs sont localisés principalement au niveau des chaînes de montagnes les plus emblématiques du Maroc : le Rif, le Moyen Atlas central et oriental et le Haut Atlas oriental (figure 1a), sur une surface estimée à 133000 ha (figure 1b). Cette espèce occupe au Maroc une tranche altitudinale moyenne comprise entre 1400 et 2400 m (Benabid, 1994) et les limites inférieure et supérieure du cèdre varient selon les régions (Pujos, 1964). Toutefois, la limite inférieure peut descendre à des niveaux encore plus bas (certains talwegs), là où des microclimats locaux sont plus humides et des expositions favorables sont rencontrées (Benabid, 1994).

L'amplitude écologique du cèdre est relativement large. II s'observe dans les bioclimats subhumide, humide et perhumide, dans leurs variantes fraîche, froide et très froide depuis l'étage supraméditerranéen jusqu'à l'oroméditerranéen, en passant par le montagnard méditerranéen (Benabid, 1994 ; M’hirit et Benzyane, 2006). Ses exigences bioclimatiques ont été décrites pour la première fois par Emberger (1938) et précisées ensuite par d'autres auteurs (Boudy, 1950 ; Pujos, 1964 ; Lecompte, 1969). Son écophysiologie correspond à des climats doux (Aussenac, 1984), même s'il est capable de supporter sans dommage des froids hivernaux importants (Benabid, 1994). Les cédraies s'observent entre des zones recevant une quantité de pluie avoisinant les $500 \mathrm{~mm}$ par an, et d'autres soumises à des précipitations qui peuvent atteindre $2000 \mathrm{~mm}$ par an. Les moyennes des minimums des températures du mois le plus froid se situent entre $-1{ }^{\circ} \mathrm{C}$ et $-8^{\circ} \mathrm{C}$ (Benabid, 1994). Cette espèce dispose d'un système racinaire très étendu, ramifié et pivotant et affiche une indifférence vis-à-vis de la nature lithologique du sol et à ses propriétés chimiques (Boudy, 1950).

\section{Approche de modélisation et outils préconisés}

La modélisation de la distribution des espèces (SDM) représente une démarche de prédiction en écologie de la conservation (Guisan et Zimmermann, 2000 ; Elith et al., 2006 ; Phillips et al., 2006 ; Austin, 2007 ; Franklin et Miller, 2010 ; Guisan et al., 2017). Elle consiste à construire une fonction de paramètres environnementaux qui permet d'observer les types de relations qui lient une espèce avec des conditions environnementales biotiques et abiotiques et de prédire par la suite la probabilité de présence de l'espèce étudiée. Aujourd'hui, ce type de modélisation est de plus en plus utilisé pour répondre à des problématiques majeures que sont la description et la prédiction de l'aire de distribution potentielle d'une espèce, et l'identification des facteurs déterminant sa distribution (Kumar et Stohlgren, 2009 ; Moukrim et al., 2019b). Plusieurs méthodes, allant du dire d'expert aux modèles mécanistes ou corrélatifs relevant de la statistique classique et/ou de l'intelligence artificielle, ont été proposées pour modéliser la distribution des espèces (Phillips et al., 2006 ; Franklin et Miller, 2010 ; Guisan et al.,
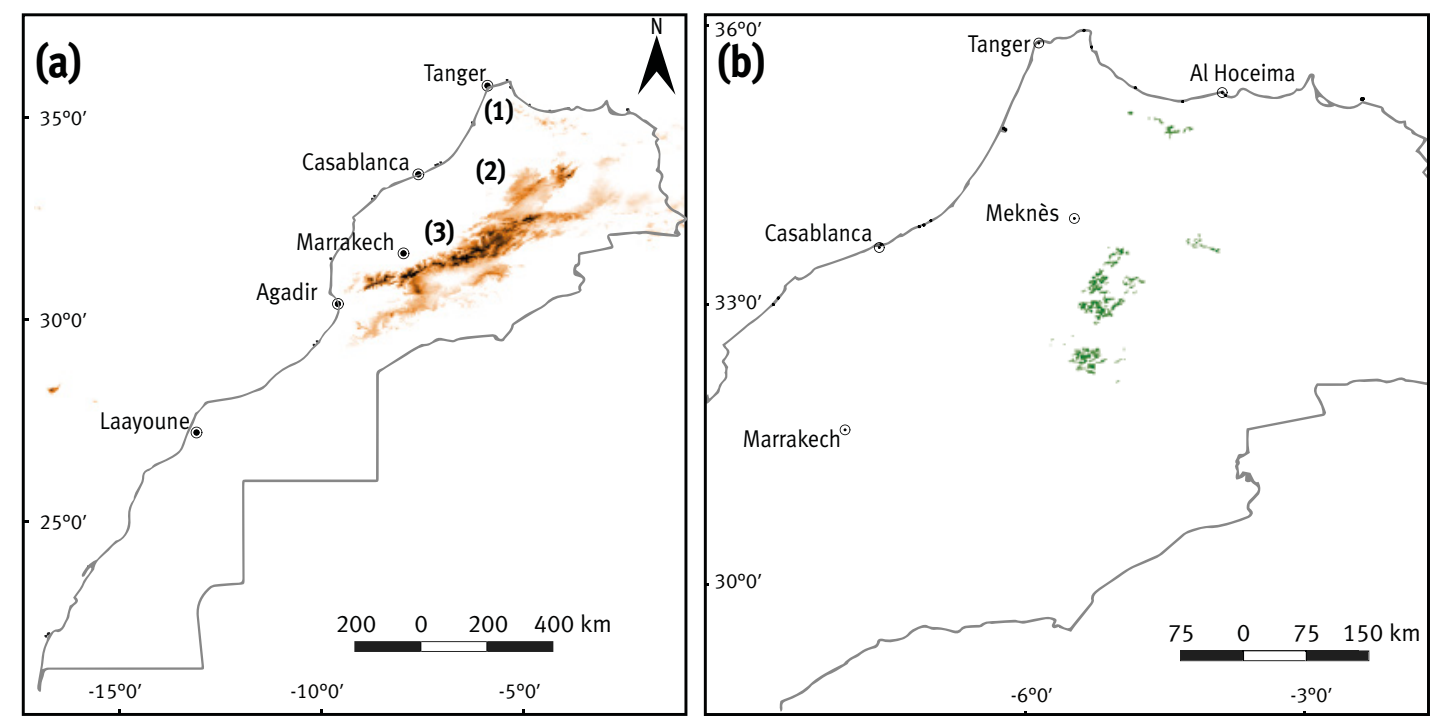

Figure 1.

Zone d'étude et occurrence réelle du cèdre. (a) Carte du Maroc avec la localisation des principales chaînes montagneuses « (1) : chaînes du Rif ; (2) ; chaînes du Moyen Atlas ; (3) : chaînes du Haut Atlas ». (b) Zoom sur les zones d'occurrence actuelle du cèdre selon l'IFN (2005). 
2017). Dans notre étude, l'approche d'entropie maximale, relevant de l'apprentissage automatique et qui trouve ses origines dans la théorie de l'information, a été adoptée, et ce via l'algorithme "MaxEnt », dans sa version 3.3.3k (Phillips et al., 2006). Cet algorithme a été choisi car il constitue l'une des méthodes pertinentes pour ce genre de modélisation (dite empirique ou corrélative) (Elith et al., 2006), permettant de générer des modèles de distribution d'espèces fiables (Baldwin, 2009). Il est spécifiquement développé pour une utilisation avec des données de présence seulement et ne nécessite que peu de points de présence de l'espèce pour construire des modèles performants (Hernandez et al., 2006 ; Phillips et al., 2006 ; Wisz et al., 2008 ; Feeley et Silman, 2011). Aussi, il est relativement insensible aux différents biais spatiaux, essentiellement liés aux méthodologies d'identification des points de présence (Elith et al., 2006 ; Graham et al., 2007).

\section{Variables utilisées}

Le jeu de données utilisé dans la présente étude comprend des données d'occurrence actuelle de l'espèce et des valeurs de certains paramètres environnementaux, qui agissent directement sur la répartition et la distribution de l'espèce étudiée. Ainsi, trois cents points d'occurrence géoréférencés du cèdre ont été échantillonnés de manière aléatoire à partir de la base de données de l'Inventaire Forestier National (IFN, 2005). Ces données ont été examinées, validées et complétées par un échantillon supplémentaire d'une vingtaine de points de présence, issus des cartes des types de peuplements réalisées dans les études d'aménagement et des relevés lors des sorties sur le terrain. Les points d'occurrence ont été séparés par une distance minimale de $1 \mathrm{~km}$, afin de n'avoir qu'un seul point de présence par pixel et limiter ainsi le biais d'échantillonnage et l'effet de l'autocorrélation spatiale dans ce genre de sondage (Guisan et Zimmermann, 2000). Vingt variables environnementales ont été sélectionnées pour cette modélisation, à savoir : 19 variables bioclimatiques qui sont les plus directement reliées aux aspects physiologiques de la croissance des plantes, pouvant limiter leurs distributions et indiquant des conditions moyennes, saisonnières et extrêmes des paramètres climatiques (Hijmans et al., 2005) ; l'altitude, documentée comme ayant une part d'influence sur la distribution de l'espèce étudiée
(Benabid, 1994).

Les données relatives aux variables environnementales sous les conditions climatiques passées (Holocène moyen), actuelles et pour les projections climatiques futures (2050 et 2070 : représentant respectivement la moyenne pour les périodes 2041-2060 et 2061-2080), proviennent de la plateforme Worldclim dans sa version 1.4 (Hijmans et al., 2005). Les données de la variable altitude proviennent, elles, du modèle numérique de terrain GTOPO30. Ces données ont été téléchargées, vérifiées et préparées à la même emprise et à la même résolution spatiale (30 secondes d'arc $\sim 1 \mathrm{~km}$ ).

Pour la simulation des aires potentielles futures, deux scénarios d'émission de gaz à effet de serre (dits optimiste RCP 2.6 et pessimiste RCP 8.5) et 18 modèles de circulation générale (GCM pour Global Circulation Model), validés par le Groupe d'experts intergouvernemental sur l'évolution du climat (GIEC) (IPCC, 2013), ont été retenus (Hijmans et al., 2005). L'utilisation de la moyenne de ces 18 GCM basés sur plusieurs combinaisons de paramètres réalisables plutôt que sur un seul modèle a été adoptée afin de limiter les incertitudes et de tirer des déductions de façon plus pertinente (Knutti et al., 2010 ; Moukrim et al., 2019b).

\section{Entraînement, validation du modèle et analyse des résultats}

Durant la simulation de la distribution du cèdre, les paramètres par défaut de l'algorithme MaxEnt, relatifs au nombre maximum de points de pseudo-absence (background data) générés par défaut par le modèle, au nombre maximum d'itérations, au seuil de convergence, et à l'ajustement du multi-

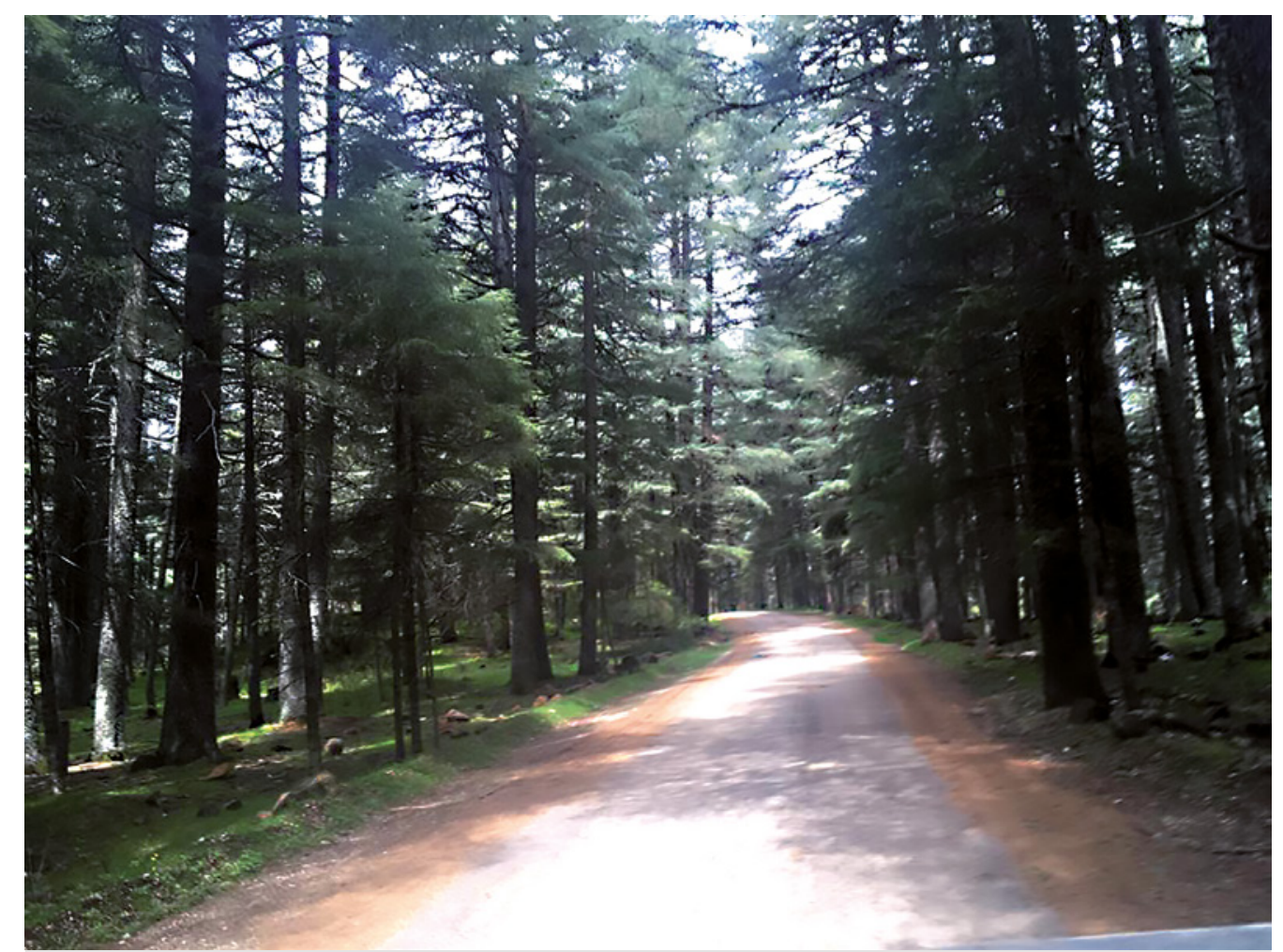

Photo 3.

Une route secondaire dans le royaume du cèdre desservant les populations riveraines et facilitant les interventions de protection dans la cédraie. La régénération naturelle de ce peuplement y est absente car il est régulièrement pâturé. Photo S. Lahssini. 
plicateur des paramètres de régularisation, ont été maintenus (Phillips et Dudík, 2008). Toutefois, les nouvelles recommandations concernant d'autres aspects (Phillips et al., 2017), tels que le format de sortie pour l'estimation des probabilités, les tests de qualité prédictive du modèle et d'identification des variables, ont été prises en considération. Ensuite, le modèle a été implémenté par les données de présence de l'espèce et des variables environnementales. La sortie brute de MaxEnt est une fonction exponentielle qui assigne une probabilité d'occurrence à chaque site (Phillips et al., 2006), nommée aussi index d'adéquation relative de l'habitat (Index of relative habitat suitability) (Fitzpatrick et al., 2013). Les valeurs brutes sont converties en une probabilité cumulative pour pouvoir égaler le chiffre 1 (Phillips et al., 2006).

Le premier test de validation des prédictions des SDM a été la validation croisée, qui permet d'examiner la variabilité dans la construction du modèle, en utilisant une partie des données (25\%) pour la calibration (Guisan et Zimmermann, 2000). La validité intrinsèque du modèle est aussi caractérisée par les paramètres sensibilité et spécificité de la courbe caractéristique d'opération, dite ROC (Receiving Operator Characteristic) (Hanley et McNeil, 1982). Le calcul de l'aire sous la courbe, dite AUC (Area Under the Curve), qui est un seuil qui mesure la précision de la prédiction du modèle, renseigne sur la performance et la qualité du modèle établi à prédire correctement l'occurrence de l'espèce (Hanley et McNeil, 1982). Les valeurs de l'AUC sont interprétées comme proposé par Swets (1988) : le modèle est « mauvais » si AUC $\leq 0,70$; « passable » si 0,70 <AUC $\leq 0,90$; « excellent » si AUC > 0,90. La qualité du modèle a également été évaluée en examinant visuellement à quel point les résultats de la modélisation correspondent aux enregistrements de présence effective de l'espèce étudiée.

Les résultats de la modélisation avec MaxEnt comprennent des évaluations de la performance du modèle et de la contribution de chaque variable au modèle (Phillips et al., 2006). Cet algorithme facilite la réalisation des courbes de réponse des différentes variables et l'opérationnalisation du test du couteau suisse, dit Jackknife (Miller, 1974), qui explore les pouvoirs prédictifs des variables considérées. La combinaison de ces différentes sorties a permis de déterminer le pouvoir prédictif de chaque variable et d'identifier celles qui contribuent le plus au modèle.

Pour une meilleure caractérisation de la distribution potentielle de l'espèce étudiée, un seuil « $S$ » est requis pour transformer les probabilités continues d'occurrence en valeurs binaires de présence/absence (Phillips et Dudík, 2008). Comme suggéré par Phillips et Dudik (2008), le seuil retenu est celui relatif à une présence d'entraînement au $10^{\mathrm{e}}$ centile, représentant la probabilité que $90 \%$ des points de présence tombent dans l'aire potentielle définie. Toutefois, l'utilisation d'une seule valeur de seuil donne un aperçu réduit de la distribution de cette espèce. De ce fait, des gradients répartis en intervalles de probabilité de présence ont été retenus.

\section{Tableau I.}

Contributions relatives et importance de la permutation (en \%) des variables utilisées dans le modèle de distribution potentielle de Cedrus alantica au Maroc.

\begin{tabular}{|l|l|c|c|} 
Variable & $\begin{array}{l}\text { Code } \\
\text { variable }\end{array}$ & $\begin{array}{c}\text { Pourcentage de } \\
\text { contribution }\end{array}$ & $\begin{array}{c}\text { Importance de } \\
\text { la permutation }\end{array}$ \\
\hline Température moyenne annuelle & Bio_01 & 0,3 & 0,3 \\
\hline Moyenne mensuelle des variations journalières & Bio_02 & 0,3 & 16,9 \\
\hline Isothermalité (Bio_02/ Bio_07) x 100 & Bio_03 & 2,4 & 0 \\
\hline Saisonnalité de température (écart-type x 100) & Bio_04 & 1 & 0,5 \\
\hline Température maximale du mois le plus chaud & Bio_05 & 0,4 & 0,2 \\
\hline Température minimale du mois le plus froid & Bio_06 & 0,3 & 0,2 \\
\hline Écart annuel de température (Bio_05 - Bio_06) & Bio_07 & 1 & 0 \\
\hline Température moyenne du trimestre le plus humide & Bio_08 & 1,4 & 0,6 \\
\hline Température moyenne du trimestre le plus sec & Bio_09 & 1,1 & 0 \\
\hline Température moyenne du trimestre le plus chaud & Bio_10 & 7,7 & 0 \\
\hline Température moyenne du trimestre le plus froid & Bio_11 & 0 & 0 \\
\hline Précipitations annuelles & Bio_12 & 6,2 & 3,5 \\
\hline Précipitations du mois le plus humide & Bio_13 & 2,1 & 31,1 \\
\hline Précipitations du mois le plus sec & Bio_14 & 24,8 & 11 \\
\hline Saisonnalité des précipitations (coefficient de variation) & Bio_15 & 0,1 & 0,4 \\
\hline Précipitations du trimestre le plus humide & Bio_16 & 0,9 & 7,3 \\
\hline Précipitations du trimestre le plus sec & Bio_17 & 44,2 & 0 \\
\hline Précipitations du trimestre le plus chaud & Bio_18 & 2,2 & 0 \\
\hline Précipitations du trimestre le plus froid & Bio_19 & 0,1 & 0,3 \\
\hline Altitude & Alt & 3,7 & 27,6 \\
\hline
\end{tabular}


Ainsi, les cartes de la distribution potentielle du cèdre ont été produites en se basant sur une classification des résultats en trois intervalles de probabilité de présence " $P$ », définis principalement par le seuil « $S$ » retenu : habitats hautement favorables pour des valeurs de « $P$ » supérieures au seuil « $S$ » $\mathrm{P} \geq \mathrm{S}$; habitats moyennement favorables pour $1 / 2 \mathrm{~S} \leq \mathrm{P}<\mathrm{S}$; habitats inappropriés pour des valeurs de $\mathrm{P}<1 / 2 \mathrm{~S}$.

La superposition de la carte des aires potentielles, où les conditions climatiques actuelles sont les plus favorables $(P \geq S)$, avec l'aire de répartition réalisée (dont l'occurrence est confirmée) a permis de comparer l'étendue des zones où l'espèce est réellement présente avec les zones prédites où l'habitat est hautement approprié. Pour apprécier le changement pour les horizons temporels futurs, des cartes de changement des aires potentielles de distribution ont été élaborées.

\section{Résultats et discussion}

\section{Qualité prédictive du modèle et variables influentes sur la distribution du cèdre}

Les résultats relatifs à la précision de la prédiction du modèle ont montré que les valeurs de l'aire sous la courbe (AUC) sont de l'ordre de 0,988 et 0,987, respectivement pour la mise en œuvre du modèle MaxEnt et pour son test. Ceci atteste l'excellente performance de cet algorithme (Swets, 1988) dans la prédiction de l'aire favorable à l'espèce étudiée.

L'analyse des résultats des pourcentages de contribution des différentes variables, de l'importance de la permutation entre variables (tableau I) et des résultats des courbes de réponse a montré que les variables qui contribuent le plus dans l'explication de la distribution de cette espèce sont les précipitations du trimestre le plus sec et les précipitations du mois le plus humide. L'analyse des résultats du test Jackknife (figure 2) a confirmé l'importance de la variable «précipitations du trimestre le plus sec » dans la modélisation de la distribution du cèdre, en suggérant que ce prédicteur environnemental présente le meilleur gain et semble avoir l'information la plus utile lorsqu'il est utilisé isolément dans le modèle. Les résultats de ce test (figure 2) ont suggéré aussi que la variable « moyenne mensuelle des variations journalières de la température » est celle qui réduit le plus le gain quand elle est omise, ce qui semble procurer au modèle des informations qui ne sont pas présentes dans les autres variables.

Les résultats de l'analyse de la contribution des différentes variables dans la modélisation de la distribution du cèdre semblent être cohérents avec l'écologie de cette espèce. Ils confirment la plage climatique du cèdre de l'Atlas, qui est caractérisée par des précipitations abondantes (durant les mois humides et même secs) et des amplitudes thermiques considérables (Emberger, 1938 ; Boudy, 1950 ; Aussenac, 1984 ; Benabid, 1994).

\section{Aire potentielle du cèdre sous les conditions climatiques actuelles}

Les résultats du modèle de distribution du cèdre ont donné lieu à des cartes de probabilité continue montrant les aires potentielles de distribution de $C$. atlantica Manetti au Maroc, pour les différents horizons temporels retenus (passé, actuel et futurs). Sous les conditions climatiques actuelles, les répartitions potentielles du cèdre sont majoritairement circonscrites dans les zones où l'espèce est actuellement présente. Sa présence est prédite au niveau du Moyen Atlas central et oriental, du Rif et du Haut Atlas oriental (figure 3a). Cette prédiction de la distribution confirme l'affinité de cette espèce pour ces régions géographiques et concorde amplement avec la littérature existante (Benabid, 1994), les données des études d'aménagement des forêts et les données de l'Inventaire forestier national (IFN, 2005). Ce modèle prédit une aire large comme hautement favorable $(P \geq S)$ à l'occurrence du cèdre sous les conditions climatiques actuelles (soit 662653 ha ; tableau II). Cette aire potentielle est répartie entre les parallèles $32^{\circ} 21^{\prime}-35^{\circ} 24^{\prime} \mathrm{N}$ et les méridiens $03^{\circ} 42^{\prime}-05^{\circ} 48^{\prime} \mathrm{O}$ (figure $3 \mathrm{a}$ ). Les fortes probabilités de présence prédites, sous les conditions climatiques actuelles, dans le Moyen Atlas central et oriental et le Rif indiquent la présence des conditions environnementales les plus favorables à cette espèce, ce qui concorde avec l'occurrence actuelle de l'espèce (figure $1 \mathrm{~b}$ ). Toutefois, le modèle prévoit des aires qui enregistrent une aptitude moyennement favo-

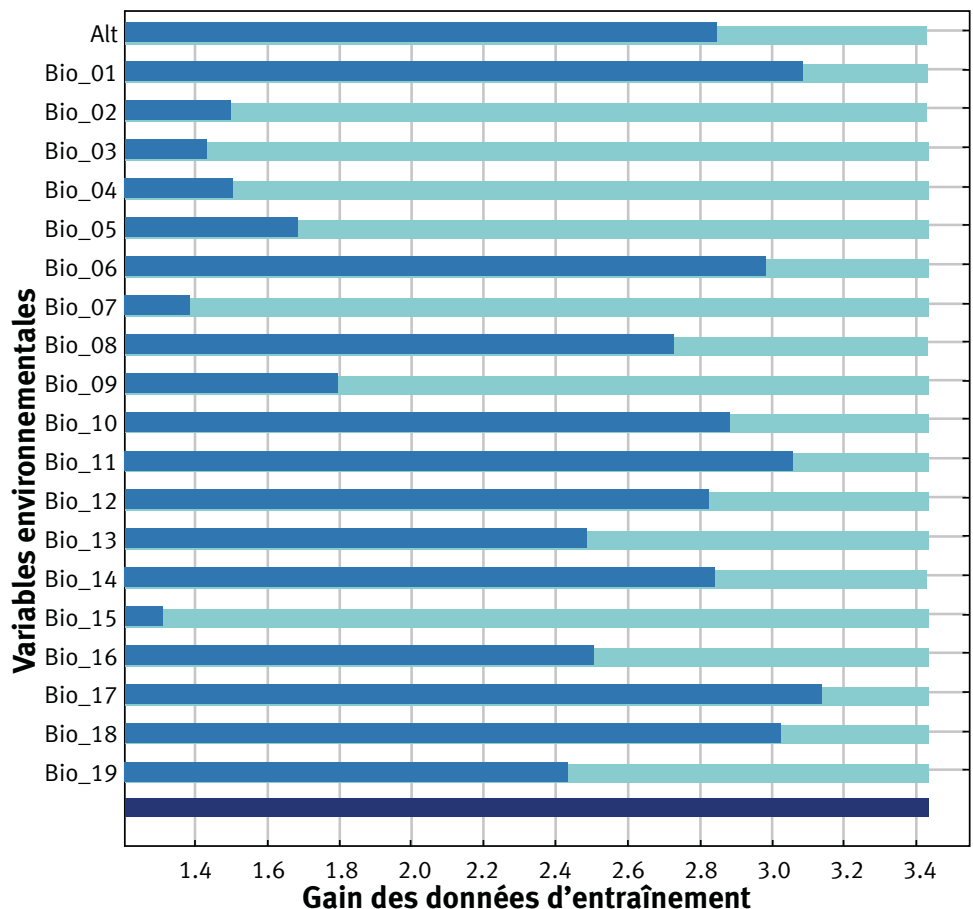

Sans la variable $\quad$ Avec la variable

Toutes les variables

Figure 2.

Résultats du test Jackknife sur la contribution des variables sélectionnées à la prédiction des aires de distribution de Cedrus atlantica. Pour chaque variable (signification au niveau du tableau I), la barre turquoise montre de combien le gain total est diminué si cette variable spécifique est exclue de l'analyse. La barre bleue montre le gain obtenu si cette variable est utilisée isolément et les variables restantes sont exclues de l'analyse. La barre bleue foncée représente le gain obtenu en introduisant toutes les variables. 
rable au cèdre dans le Haut Atlas oriental alors que l'espèce est actuellement présente dans ces zones. Une hypothèse est que ces zones, caractérisées actuellement par des difficultés de régénération, représentent une limite de l'aire de répartition de cette espèce sous les conditions climatiques actuelles, et les dépérissements accentués, parfois inexpliqués (M'hirit et Benzyane, 2006), des peuplements dans ces aires peuvent être causés par le changement climatique qui s'opère actuellement.

Les prédictions relatives aux habitats moyennement appropriés de la partie sud-ouest du Haut Atlas (sud-est de la ville de Marrakech) indiquent un environnement moyennement favorable (figure 3a), mais l'espèce n'y est pas présente réellement (figure $1 \mathrm{~b}$ ), sauf dans un périmètre situé

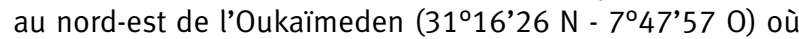
l'essai d'installation du cèdre a enregistré un taux de réussite satisfaisant (Guedira et al., 2008). Une hypothèse est que les décisions prises dans le passé pour la restauration des écosystèmes forestiers étaient en faveur des espèces autochtones, déjà présentes dans cette région (thuya, genévrier, chêne vert, etc.), sans tenir compte de l'évolution historique du cortège forestier.
La comparaison des aires actuelles de distribution, potentielle et effective, ont montré que la totalité de l'aire réelle du cèdre (figure $1 \mathrm{~b}$ ) est prédite comme hautement ou moyennement appropriée à cette espèce. La majorité des mailles (pixels) d'occurrence de cette espèce (72\%) concordent avec les aires hautement favorables, à l'exception de quelques zones circonscrites au sud de l'aire d'occurrence actuelle (figure $3 a$ ) et qui représentent des espaces où le cèdre est réellement présent, mais classées par le modèle développé comme moyennement appropriées. Aussi, cette superposition fait ressortir que l'aire potentielle prédite, sous les conditions climatiques actuelles, comme hautement favorable est largement supérieure (662 653 ha) (figure 3a) à l'aire de répartition actuelle du cèdre (133000 ha) (figure 1b). En effet, les conditions peuvent être favorables à la présence du cèdre, mais ce dernier peut ne pas être présent, du fait de la concurrence interspécifique, de ses capacités de dissémination, ou des choix sylvicoles opérés dans le passé. Ainsi, cette espèce est, par exemple, quasi absente dans certaines parties du Moyen Atlas, principalement aux alentours (parties nordiques) des aires de distribution actuelles, où les conditions écolo-

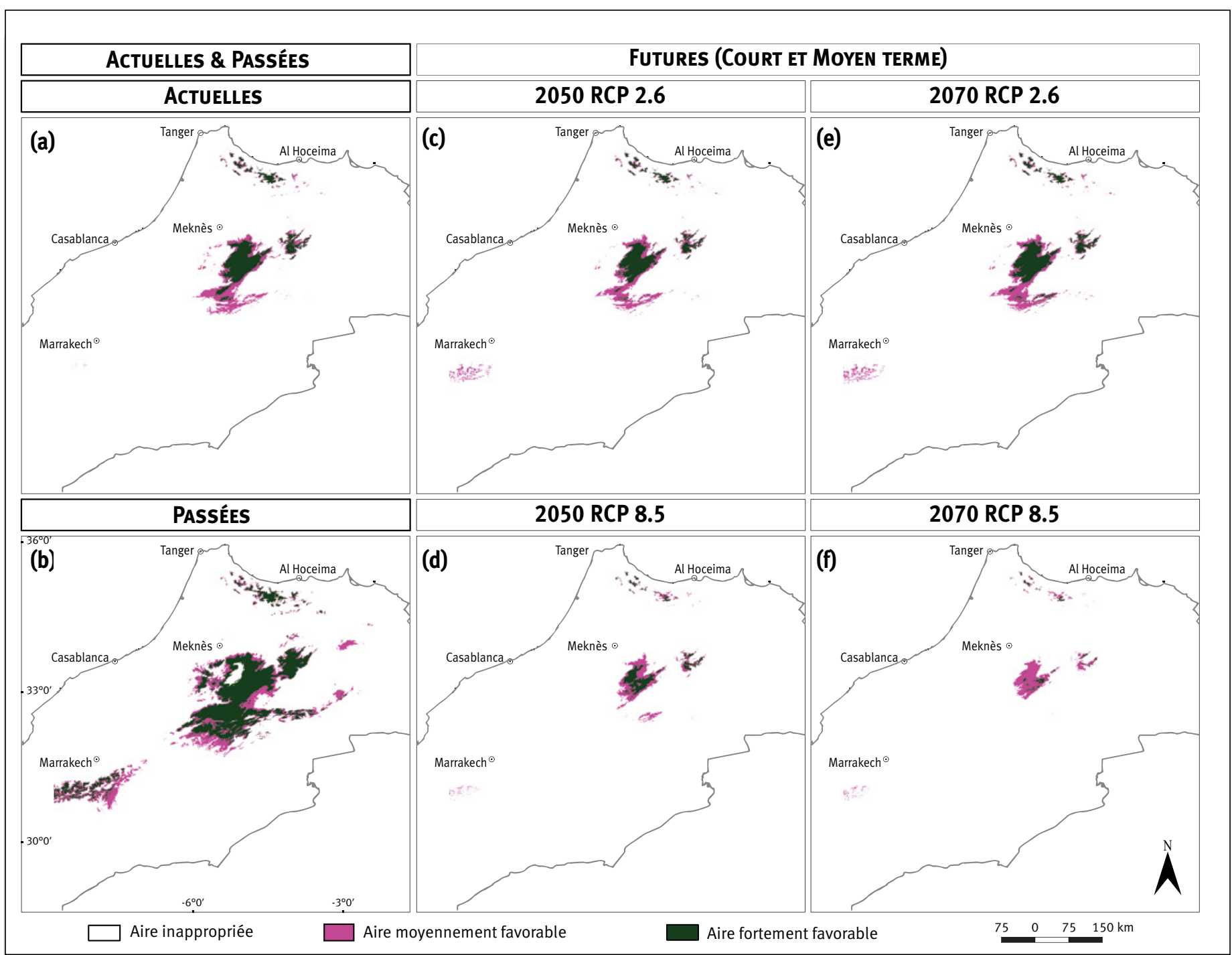

Figure 3.

Cartes des probabilités de présence montrant les aires potentielles favorables au cèdre sous les conditions climatiques actuelles (a), passées (b) et futures; pour le court terme 2050 : RCP 2.6 (c), RCP 8.5 (d) ; pour le moyen terme 2070 : RCP 2.6 (e), RCP 8.5 (f) (avec RCP 2.6 : scénario du changement climatique optimiste ; RCP 8.5 : scénario du changement climatique pessimiste). 
giques sont hautement favorables. Ceci est probablement le fait des difficultés rencontrées dans sa régénération et les décisions prises dans le passé récent pour l'extension de certaines espèces (pins, cyprès, parfois le chêne vert) pour subvenir aux besoins des populations locales en matière de bois de chauffage dans ces zones. Cette absence du cèdre peut aussi être due aux exploitations abusives opérées dans le passé (durant la période coloniale) et aux coupes illicites, sachant que le bois du cèdre est fortement recherché.

\section{Aire potentielle du cèdre sous les conditions climatiques passées}

Sous les conditions climatiques passées, précisément durant l'Holocène moyen, notre modèle a prédit des aires hautement favorables au cèdre sur de larges surfaces dans le Moyen Atlas central et oriental, au Haut Atlas oriental et au Rif (figure $3 b$ ), ce qui confirme l'affinité de cette espèce pour ces parties du Maroc. En outre, le modèle développé a montré que d'autres zones étaient propices au cèdre, avec des probabilités moyennes à élevées et sur des superficies importantes, essentiellement dans la partie sud-ouest du Haut Atlas. Ces résultats étayent les études antérieures évoquant la résurgence du cèdre au Maroc pendant l'Holocène. En effet, certaines études paléo-écologiques renseignent la présence du cèdre dans la région nord-africaine et ce depuis 8000 ans avant le présent, pour former de vrais écosystèmes forestiers et dominer le paysage de certaines zones spécifiques, notamment dans le Moyen Atlas et le Rif vers 4500 ans avant le présent (Lamb et van der Kaars, 1995 ; Cheddadi et al., 2016). Certaines études ont également révélé, en se basant sur les études polliniques (Reille, 1976), la présence de pollen de cèdre dans un passé récent (1 000 à 2000 ans avant le présent), mais à faible pourcentage, au niveau du Haut Atlas oriental et central. Ces résultats semblent confirmer la probabilité de présence des conditions environnementales favorables à cette espèce dans le passé dans ces zones géographiques et montrent ainsi que le changement climatique associé aux actions anthropiques a engendré une détérioration de ces conditions appropriées à cette espèce dans le sud-ouest du Haut Atlas. Ceci pourrait expliquer, d'une part, l'aptitude actuelle de ces milieux qui ont été définis par le modèle comme moyennement favorables à l'espèce et, d'autre part, l'actuelle absence de cette espèce au sein de cette zone qui était favorable dans un passé récent (figure 3b).

La comparaison des aires potentielles hautement favorables au cèdre sous les conditions climatiques actuelles et passées a fait ressortir que l'aire potentielle passée (figure $3 b$ ) était beaucoup plus étendue que l'aire potentielle actuelle (figure $3 a$ ). Ainsi, le modèle révèle la sensibilité du cèdre aux changements climatiques passés, et ce en accord avec les données paléo-écologiques.

\section{Aire potentielle du cèdre sous les conditions climatiques futures}

L'analyse des résultats des figures $3 c$ à $3 f$ montre que les distributions potentielles du cèdre, prédites par le modèle MaxEnt à court terme (2050, figures $3 c$ et $3 d$ ) et à moyen terme (2070, figures 3e et 3f), sont localisées dans certains sites spécifiques et semblent dépendre fortement des projections climatiques futures. Les aires potentielles futures risquent substantiellement d'être affectées négativement par le changement climatique. L'analyse de cette figure montre que les aires potentielles hautement favorables pour le cèdre diminueront dans les horizons futurs, ceci pour les deux scénarios climatiques. Ces diminutions, calculées en comparant les distributions potentielles futures et actuelles, peuvent aller de $-21,7 \%$ sous le scénario optimiste RCP 2.6 (à l'horizon 2050) à - 93,1 \% sous le scénario pessimiste RCP 8.5 (à l'horizon 2070) (tableau II). Les pertes enregistrées dans la classe hautement favorable seront compensées en partie par des gains dans la classe moyennement favorable (gains allant de $40 \%$ à $130 \%$ par rapport à l'aire potentielle moyennement favorable sous les conditions actuelles), ce qui explique certaines améliorations de l'aire favorable totale sous le scénario optimiste pour les deux horizons temporels (tableau II).

Sur le plan de la répartition zonale des pertes, les cédraies du Moyen Atlas central et oriental et du Haut Atlas oriental seront les plus touchées par le changement climatique futur et connaîtront les plus fortes régressions compa-
Tableau II.

Aire potentielle de Cedrus atlantica au Maroc sous les conditions climatiques futures.

\begin{tabular}{|c|c|c|c|}
\hline \multirow[b]{3}{*}{ Aire réelle du cèdre } & \multicolumn{3}{|c|}{ Aire potentielle favorable (ha) } \\
\hline & Total & $\begin{array}{l}\text { Hautement } \\
\text { favorable }\end{array}$ & $\begin{array}{l}\text { Moyennement } \\
\text { favorable }\end{array}$ \\
\hline & 133000 & & \\
\hline Aire potentielle actuelle (ha) & 961510 & 662653 & 298857 \\
\hline \multicolumn{4}{|l|}{ Prédictions futures } \\
\hline \multicolumn{4}{|l|}{2050} \\
\hline RCP2.6 & $1179470(22,7)$ & $519188(-21,7)$ & $660282(120,9)$ \\
\hline RCP8.5 & $675055(-29,8)$ & $243162(-63,3)$ & $431893(44,5)$ \\
\hline \multicolumn{4}{|l|}{2070} \\
\hline RCP2.6 & $1283039(33,4)$ & $595739(-10,1)$ & $687300(130)$ \\
\hline RCP8.5 & $465942(-51,5)$ & $45978(-93,1)$ & $419964(40,5)$ \\
\hline
\end{tabular}

Les pourcentages des changements des aires favorables potentielles sous les conditions climatiques futures par rapport aux aires potentielles actuelles sont donnés entre parenthèses. RCP représente les scénarios des changements climatiques (RCP2.6 : scénario optimiste ; RCP8.5 : scénario pessimiste). 
rativement au Rif. On note également que le modèle a pu prédire la présence du cèdre, avec des probabilités moyennement favorables, dans la partie sud-ouest du Haut Atlas sous les conditions climatiques futures (figures $3 c$ et $3 f$ ), bien que cette espèce soit actuellement absente dans cette zone (figure $1 \mathrm{~b}$ ). Ceci signifie que les conditions environnementales dans ces zones seront plus ou moins favorables au développement de cette espèce dans le futur.

\section{Impact du changement climatique sur l'aire potentielle}

La comparaison des cartes de distribution potentielle sous les conditions climatiques actuelles et futures a permis d'estimer l'impact du changement climatique sur la répartition du cèdre. Les résultats de cette comparaison indiquent que l'aptitude des espaces au développement de cette espèce connaîtra des variations dans le futur (figures 4). Des réductions dans certaines régions, et des expansions dans d'autres, de la gamme favorable sont projetées dans le futur (tableau III). Pour les deux horizons temporels et sous les deux scénarios des changements climatiques retenus, les modèles prédisent une perte importante des aires hautement favorables à cette espèce. Le pourcentage de variation des potentialités du milieu au développement de cette espèce indique des valeurs négatives pour tous les scénarios, allant de - 23,1 \% à - $94 \%$. Les plus fortes régressions de l'aire hautement favorable sont prévues sous le scénario RCP 8.5 à l'horizon 2070 (tableau III). Ces résultats sont en accord avec ceux issus d'une autre étude indiquant que la distribution de l'espèce étudiée était plus large durant le siècle dernier (Boudy, 1950)

Les gains d'aire de distribution dans le futur resteront limités et ne dépasseront guère les 5,3\% de l'aire actuelle hautement favorable (tableau III), soit une superficie de
34997 ha à moyen terme, d'après le scénario RCP 2.6. Ces gains seront enregistrés essentiellement dans les parties orientales du Haut Atlas et du Moyen Atlas et la partie nordique du Rif, et ce au sein des zones limitrophes de l'aire potentielle définie sous les conditions climatiques actuelles. Ces dernières zones étant caractérisées par des altitudes plus importantes (figures 4), ceci pourrait s'expliquer par l'interdépendance entre les paramètres climatiques et les facteurs géographiques tels que l'altitude et pourrait témoigner d'une possible migration latitudinale et altitudinale de l'espèce vers des refuges où elle pourrait rencontrer les conditions environnementales qui resteront en adéquation avec sa niche dans le futur (Rhanem, 2011). Ces tendances de déplacements concordent avec la littérature mentionnant des mouvements latitudinaux et altitudinaux des espèces dans différentes parties du monde (Walther et al., 2002).

Les résultats de ce travail font ressortir que de nouvelles zones potentielles, même limitées, apparaîtront dans le futur. Ce qui concorde avec certaines études, qui ont reconnu que le réchauffement climatique pourrait avoir aussi un effet positif sur les écosystèmes et les espèces dans certaines parties du monde, via l'extension de leurs habitats favorables (IPCC, 2018). Toutefois, les gains prévus dans le futur ne contribueront pas à la compensation totale des pertes prédites, étant donné le fait que la colonisation de nouvelles zones par une espèce végétale dépendra fortement de sa capacité spécifique à s'adapter localement aux nouvelles conditions climatiques, des facteurs limitants de l'expansion des espèces (biologiques, écologiques et génétiques), de son utilisation, de sa gestion et des perturbations anthropiques futures (Phillips et al., 2006).

L'analyse des changements de l'aptitude du milieu montre que le changement climatique pourrait avoir des impacts, principalement négatifs, sur les aires potentielles favorables à C. atlantica dans les différentes zones de présence de cette espèce. Cela pourrait entraîner une nette régression des superficies du cèdre dans le futur, et impacter considérablement les fonctions et services de cet écosystème et augmenter les risques d'extinction locale de cette espèce dans le futur. Ces résultats confirment ceux d'autres travaux, spécifiques à cette espèce (Cheddadi et al., 2017) et/

Les pourcentages des changements des aires favorables potentielles sont donnés entre parenthèses. RCP représente les scénarios des changements climatiques (RCP2.6 : scénario optimiste ; RCP8.5 : scénario pessimiste) ; P représente la probabilité de présence ; $S$ représente le seuil retenu pour définir les aires favorables. 
ou sur d'autres espèces végétales en général, telles que Linaria nigricans et Sorbus tauricola respectivement endémiques du sud-est de l'Espagne et du sud de la Crimée en Ukraine (Thomas et al., 2004 ; Hooper et al., 2012 ; Hallegatte et al., 2015 ; IPCC, 2018). Aussi, ces variations d'aire potentielle du cèdre sous les conditions climatiques futures corroborent les résultats de plusieurs études et rapports sur les écosystèmes naturels (Hallegatte et al., 2015 ; IPCC, 2018).

\section{Implications pour la conservation de la cédraie}

Face aux impacts du changement climatique et aux incertitudes engendrées par ce phénomène, les outils classiques pour le choix des actions de conservation et de développement de la cédraie apparaissent peu adaptés pour guider les gestionnaires et les scientifiques en termes de choix des sites mais aussi en termes d'intensité d'action (choix des techniques) et de temporalité convenables. Une telle inadaptation est remarquée dans les plans de gestion actuels des différentes études d'aménagement des forêts et des aires protégées marocaines, qui considèrent rarement que la distribution spatiale naturelle des espèces cibles peut varier dans le contexte de changement climatique.

Les cartes, ainsi produites sous les conditions climatiques actuelles, passées et futures (figure 3), améliorent nos connaissances sur la biogéographie et l'écologie de l'espèce étudiée et apportent une information complémentaire par rapport aux seules données d'occurrence ou aux cartes de répartition existantes. Elles constituent un outil de réflexion à la disposition du scientifique et du gestionnaire pour l'aide à la décision et l'appréhension du comportement de cette espèce dans le contexte du chan-

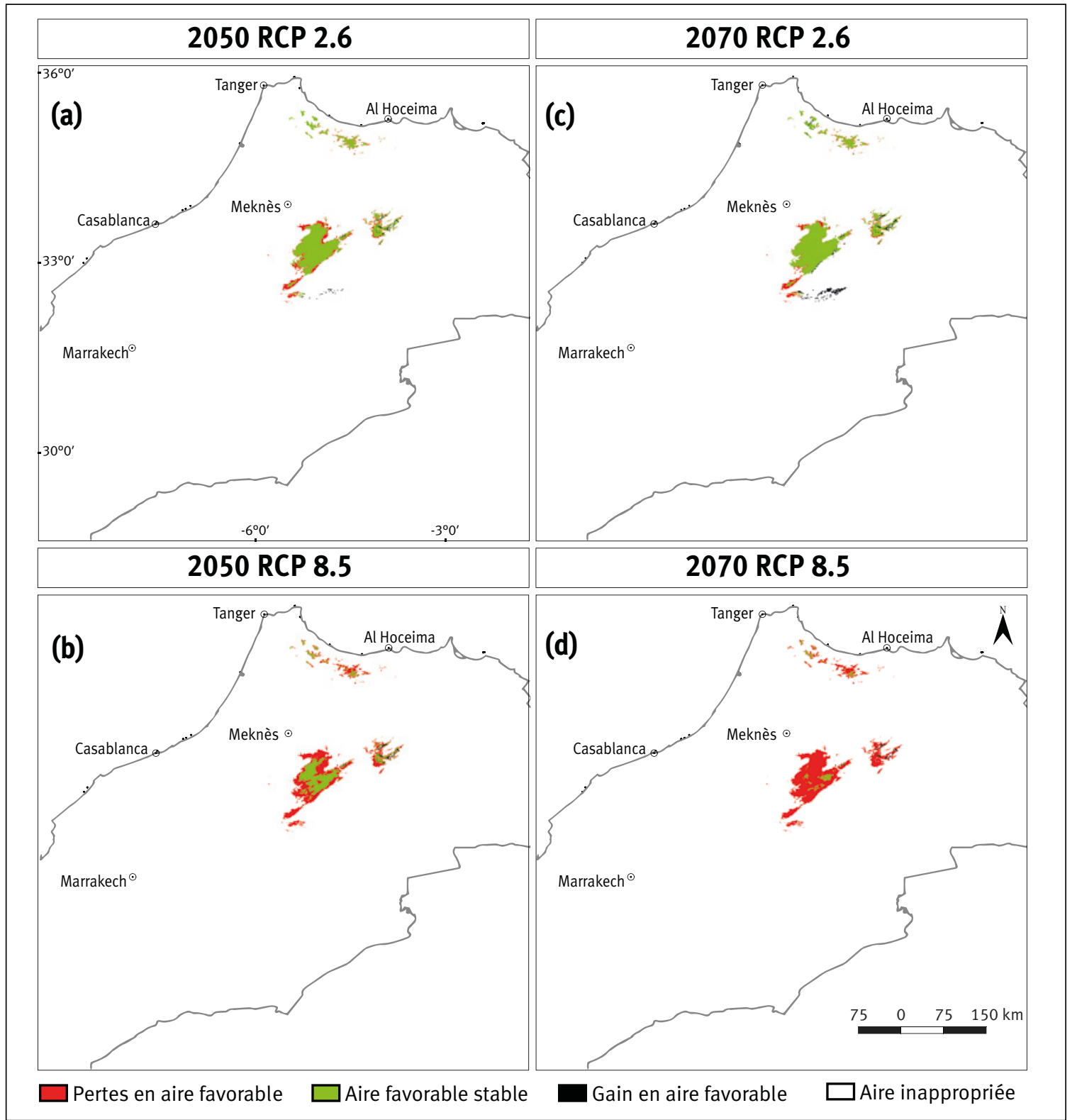

Figure 4.

Cartes des changements de l'aire potentielle hautement favorable au cèdre dans le futur et ce pour le court terme 2050 : RCP 2.6 (a), RCP 8.5 (b) ; pour le moyen terme 2070 : RCP 2.6 (c), RCP 8.5 (d) (avec RCP 2.6 : scénario du changement climatique optimiste; RCP 8.5 : scénario du changement climatique pessimiste). 
gement climatique. Ces cartes permettront d'identifier et de clarifier le choix des sites d'actions prioritaires pour la conservation et le développement du cèdre de l'Atlas. Elles permettront aussi de mieux raisonner l'intensité des interventions et de remodeler les efforts en fonction de l'aptitude et de la probabilité de présence enregistrée dans les différents milieux et des difficultés stationnaires. Des stratégies de conservation in situ et ex situ doivent ainsi être privilégiées. Les zones hautement favorables sous les conditions climatiques actuelles, principalement celles peuplées actuellement par l'espèce concernée, et qui resteront favorables à cette espèce dans le futur, doivent être gérées par des actions urgentes de conservation in situ, et ce d'une manière concertée et en impliquant la population usagère (Moukrim et al., 2019a). Les zones prédites comme hautement favorables sous les conditions climatiques actuelles, mais où l'espèce n'y est pas réellement présente (absence d'occurrence réelle) et qui resteront favorables dans le futur, devront être encadrées par des programmes de régénération artificielle et d'assistance à la migration de l'espèce, du moins d'une manière expérimentale, pour la conservation et l'élargissement des zones dites de micro-refuge pour l'espèce dans le futur (Cheddadi et al., 2017).

Sur un autre registre, les cartes produites pourraient être aussi des outils importants pour la concrétisation des objectifs escomptés de la nomination de l'écosystème cédraie en réserve de biosphère, en intégrant les évolutions futures des aires potentielles dans le plan d'aménagement et de gestion de cette réserve. Également, elles peuvent être utiles pour argumenter une révision et aider à une nouvelle conception des limites de cette réserve de biosphère dans le futur.

\section{Conclusion}

Dans cette étude, la modélisation de l'aire potentielle de Cedrus atlantica Manetti au Maroc pour les différents horizons temporels (passé, actuel et les deux scénarios climatiques futurs) a été réalisée sur l'ensemble du territoire du Maroc. Les résultats de ce travail montrent que les futures zones d'habitat appropriées seront site-spécifiques et semblent être fortement tributaires des conditions climatiques futures. L'analyse des changements d'aptitude des milieux montre que le changement climatique peut avoir un impact, essentiellement négatif, sur les aires potentielles favorables à cette espèce. L'ampleur de ces changements aura des effets considérables sur les fonctions, les biens et les services rendus par cet écosystème ainsi que des impacts significatifs sur les zones de conservation et de gestion actuelles.

Ces résultats soulèvent néanmoins d'importantes questions concernant la gestion de la biodiversité en général et des écosystèmes forestiers naturels en particulier, à la lumière des changements climatiques. Par conséquent, les scénarios climatiques futurs doivent être inclus dans les stratégies de conservation existantes pour la protection des espèces forestières et des biens et services qu'elles procurent, et ce pour éviter toute future extinction locale, sachant que les écosystèmes forestiers marocains abritent une part importante de la biodiversité nationale.

\section{Remerciements}

Nous remercions les trois évaluateurs anonymes pour leurs observations, remarques et suggestions pertinentes qui ont amélioré la qualité du manuscrit. Nos remerciements vont aussi aux collègues gestionnaires du département des Eaux et Forêts avec qui nous avons eu des discussions utiles.

\section{Références bibliographiques}

Aussenac G., 1984. Le Cèdre, essai d'interprétation bioclimatique et écophysiologique. Bulletin de la Société Botanique de France. Actualités Botaniques, 131 (2-4) : 385-398. https://doi.org/10.1080/01811789.19 84.10826679

Austin M., 2007. Species distribution models and ecological theory: A critical assessment and some possible new approaches. Ecological Modelling, 200 (1-2): 1-19. https://doi.org/10.1016/j. ecolmodel.2006.07.005

Baldwin R. A., 2009. Use of Maximum Entropy Modeling in Wildlife Research. Entropy, 11 (4): 854-866. https://doi.org/10.3390/e11040854

Photo 4.

Façonnant le paysage, de beaux cèdres jalonnent la route nationale RN 13.

Photo S. Lahssini. 
Benabid A., 1994. Biogéographie, phytosociologie et phytodynamique des cédraies de l'Atlas Cedrus atlantica (Manetti). Annales de la Recherche Forestière au Maroc, 27 : 33-60.

Boudy P., 1950. Économie forestière nord-africaine. Tome 2 : Monographies et traitements des essences forestières. Paris, France, Larose, 878 p.

Cheddadi R., Bouaissa O., Rhoujjati A., Dezileau L., 2016. Environmental changes in the Moroccan western Rif mountains over the last 9,000 years. Quaternaire, 27 (1): 15-25. https://doi.org/10.4000/quaternaire.7517

Cheddadi R., Henrot A.-J., François L., Boyer F., Bush M., Carré M., et al., 2017. Microrefugia, Climate Change, and Conservation of Cedrus atlantica in the Rif Mountains, Morocco. Frontiers in Ecology and Evolution, 5: 1-15. https://doi.org/10.3389/fevo.2017.00114

Elith J., Graham C. H., Anderson R. P., Dudík M., Ferrier S., Guisan A., Hijmans R. J., et al., 2006. Novel methods improve prediction of species' distributions from occurrence data. Ecography, 29 (2): 129 151. https://doi.org/10.1111/j.2006.0906-7590.04596.x

Emberger L., 1938. Contribution à la connaissance des cèdres et en particulier du déodar et du cèdre de l'Atlas. Revue de Botanique Appliquée et d'Agriculture Coloniale, 18 (198) : 77-92. https://doi.org/10.3406/jatba.1938.5828

Feeley K. J., Silman M. R., 2011. Keep collecting: accurate species distribution modelling requires more collections than previously thought. Diversity and Distributions, 17 (6): 1132-1140. https://doi.org/10.1111/i.1472-4642.2011.00813.x

Fennane M., Ibn Tattou M., 2012. Statistiques et commentaires sur l'inventaire actuel de la flore vasculaire du Maroc. Bulletin de l'Institut Scientifique, Rabat, section Sciences de la Vie, 34 (1) : 1-9. http://www.israbat.ac.ma/wp-content/uploads/2015/01/01Fennane BIS SV34 1 1.pdf

Fitzpatrick M. C., Gotelli N. J., Ellison A. M., 2013. MaxEnt versus MaxLike: empirical comparisons with ant species distributions. Ecosphere, 4 (5): art55. https://doi.org/10.1890/es13-00066.1

Franklin J., Miller J. A., 2010. Mapping species distributions: spatial inference and prediction. Cambridge, UK, Cambridge University Press, Coll. Ecology, biodiversity and conservation, 320 p. https://doi.org/10.1017/CB09780511810602

Gbètoho A. J., Aoudji A. K. N., Roxburgh L., Ganglo J. C., 2017. Assessing the suitability of pioneer species for secondary forest restoration in Benin in the context of global climate change. Bois et Forêts des Tropiques, 332 (2): 43-55. https://doi.org/10.19182/bft2017.332.a31332

Graham C. H., Elith J., Hijmans R. J., Guisan A., Townsend Peterson A., Loiselle B. A., et al., 2007. The influence of spatial errors in species occurrence data used in distribution models: Spatial error in occurrence data for predictive modelling. Journal of Applied Ecology, 45 (1): 239-247. https://doi.org/10.1111/i.1365-2664.2007.01408.x

Guedira A., Satrani B., Douira A., 2008. Effet de la densité de Cytisus grandiflorus (Brot.) DC. sur la fertilité du sol en azote et son influence sur la croissance d'une plantation de cèdre (Cedrus atlantica Manetti). Acta Botanica Gallica, 155 (3): 375-387. https://doi.org/10.1080/12538078.2008.10516118

Guisan A., Thuiller W., Zimmermann N. E., 2017. Habitat suitability and distribution models with applications in R. Cambridge, UK, Cambridge University Press, Coll. Ecology, biodiversity and conservation. https://doi.org/10.1017/9781139028271

Guisan A., Zimmermann N. E., 2000. Predictive habitat distribution models in ecology. Ecological Modelling, 135 (2): 147-186. https://doi.org/10.1016/s0304-3800(00)00354-9
Hallegatte S., Fay M., Bangalore M., Kane T., Bonzanigo L., 2015. Shock waves: Managing the impacts of climate change on poverty. Washington DC, USA, World Bank Publications, 227 p. https://doi.org/10.1596/978-1-4648-0673-5_fm

Hanley J. A., McNeil B. J., 1982. The meaning and use of the area under a receiver operating characteristic (ROC) curve. Radiology, 143 (1): 29-36. https://doi.org/10.1148/radiology.143.1.7063747

Hernandez P. A., Graham C. H., Master L. L., Albert D. L., 2006. The effect of sample size and species characteristics on performance of different species distribution modeling methods. Ecography, 29 (5): 773-785. https://doi.org/10.1111/j.0906-7590.2006.04700.x

Hijmans R. J., Cameron S. E., Parra J. L., Jones P. G., Jarvis A., 2005. Very high resolution interpolated climate surfaces for global land areas. International Journal of Climatology, 25 (15): 1965-1978. https://doi.org/10.1002/joc.1276

Hooper D. U., Adair E. C., Cardinale B. J., Byrnes J. E., Hungate B. A. Matulich K. L., et al., 2012. A global synthesis reveals biodiversity loss as a major driver of ecosystem change. Nature, 486 (7401): 105-108. https://doi.org/10.1038/nature11118

Hughes L., 2000. Biological consequences of global warming: is the signal already apparent? Trends in Ecology \& Evolution, 15 (2): 56 61. https://doi.org/10.1016/s0169-5347(99)01764-4

IFN, 2005. Inventaire forestier national. Rabat, Maroc, HautCommissariat aux Eaux et Forêts et à la Lutte contre la Désertification.

IPCC, 2013. Climate change 2013: the physical science basis. Contribution of working group I to the fifth assessment report of the intergovernmental panel on climate change. Cambridge, UK, Cambridge University Press, 1535 p. https://www.ipcc.ch/site/ assets/uploads/2018/03/WG1AR5 SummaryVolume FINAL.pdf

IPCC, 2018. Global warming of $1.5^{\circ} \mathrm{C}$ : An IPCC Special Report on the impacts of global warming of $1.5^{\circ} \mathrm{C}$ above pre-industrial levels and related global greenhouse gas emission pathways, in the context of strengthening the global response to the threat of climate change, sustainable development, and efforts to eradicate poverty. Cambridge, UK, Cambridge University Press, 562 p. https://www.ipcc.ch/sr15/

Knutti R., Abramowitz G., Eyring V., Gleckler P. J., Hewitson B., Mearns L., 2010. Good practice guidance paper on assessing and combining multi model climate projections. In: Stocker T., Dahe Q., Plattner G.-K., Tignor M., Midgley P. (eds.). Meeting Report of the IPCC Expert meeting on assessing and combining multi model climate projections. IPCC working group I technical support unit, Bern, Switzerland, University of Bern, 127 p. https://www.ipcc. ch/publication/ipcc-expert-meeting-on-assessing-and-combiningmulti-model-climate-projections/

Kumar S., Stohlgren T. J., 2009. Maxent modeling for predicting suitable habitat for threatened and endangered tree Canacomyrica monticola in New Caledonia. Journal of Ecology and the Natural Environment, 1 (4): 094-098. https://academicjournals.org/ journal/JENE/article-abstract/C1CDB822968

Lamb H. F., van der Kaars S., 1995. Vegetational response to Holocene climatic change: pollen and palaeolimnological data from the Middle Atlas, Morocco. The Holocene, 5 (4): 400-408. https://doi.org/10.1177/095968369500500402

Lecompte M., 1969. La végétation du moyen Atlas central : esquisse phytoécologique et carte des séries de végétation au 1: 200000. Rabat, Maroc, Institut scientifique chérifien, $34 \mathrm{p}$.

McCarty J. P., 2001. Ecological consequences of recent climate change. Conservation Biology, 15 (2): 320-331. https://doi.org/10.1046/j.1523-1739.2001.015002320.x

MDCE, 2016. La troisième communication nationale à la Conventioncadre des Nations unies sur les changements climatiques. Rabat, Maroc, ministère de l'Environnement, 132 p. http://www.un-gsp. org/sites/default/files/documents/3069145_cite_divoire-nc3-1. cote divoire - third national communication.pdf 
MEA, 2005. Millennium Ecosystem Assessment (Program): Ecosystems and human well-being: synthesis. Washington DC, USA, Island Press, 137 p. https://www.millenniumassessment.org/ documents/document.356.aspx.pdf

M'hirit O., Benzyane M., 2006. Le Cèdre de l'Atlas: Mémoire du temps. Bruxelles, Belgique, Mardaga, 288 p.

Miller R. G., 1974. The jackknife - a review. Biometrika, 61 (1): 1-15. https://doi.org/10.1093/biomet/61.1.1

Moukrim S., Lahssini S., Naggar M., Lahlaoi H., Rifai N., Arahou M., et al., 2019a. Local community involvement in forest rangeland management: case study of compensation on forest area closed to grazing in Morocco. The Rangeland Journal, 41 (1): 43-53. https://doi.org/10.1071/ri17119

Moukrim S., Lahssini S., Rhazi M., Mharzi A. H., Benabou A., Wahby I., et al., 2019b. Climate change impacts on potential distribution of multipurpose agro-forestry species: Argania spinosa (L.) Skeels as case study. Agroforestry Systems, 93 (4): 1209-1219. https://doi.org/10.1007/s10457-018-0232-8

Myers N., Mittermeier R. A., Mittermeier C. G., Da Fonseca G. A., Kent J., 2000. Biodiversity hotspots for conservation priorities. Nature, 403 (6772): 853-858. https://doi.org/10.1038/35002501

Pearson R. G., Dawson T. P., 2003. Predicting the impacts of climate change on the distribution of species: are bioclimate envelope models useful? Global Ecology and Biogeography, 12 (5): 361-371. https://doi.org/10.1046/j.1466-822x.2003.00042.X

Phillips S. J., Anderson R. P., Dudík M., Schapire R. E., Blair M. E., 2017. Opening the black box: an open-source release of Maxent. Ecography, 40: 887-893. https://doi.org/10.1111/ecog.03049

Phillips S. J., Anderson R. P., Schapire R. E., 2006. Maximum entropy modeling of species geographic distributions. Ecological Modelling, 190 (3-4): 231-259. https://doi.org/10.1016/i.ecolmodel.2005.03.026

Phillips S. J., Dudík M., 2008. Modeling of species distributions with Maxent: new extensions and a comprehensive evaluation. Ecography, 31 (2): 161-175. https://doi.org/10.1111/i.0906-7590.2008.5203.x

Pujos A., 1964. Les milieux de la cédraie marocaine. Étude d'une classification des cédraies du Moyen Atlas et du Rif en fonction du sol et du climat et de la régénération actuelle dans ces peuplements. Annales de la Recherche Forestière au Maroc, 8 : 1-283.

Rabhi K., Akli A., Djouhri A., Yahi N., Boudedja S, Messaoudene M., 2018. Bilan et croissance des reboisements de cèdre de l'Atlas, Cedrus atlantica (Endl.) Carrière, en Algérie : cas du Djurdjura et de l'Atlas blidéen. Bois et Forêts des Tropiques, 337 (3): 3-15. https://doi.org/10.19182/bft2018.337.a31627

Reille M., 1976. Analyse pollinique de sédiments postglaciaires dans le Moyen Atlas et le Haut Atlas marocains: premiers résultats. Ecologia Mediterranea, 2 : 154-172. https://doi.org/10.3406/ecmed.1976.925

Rhanem M., 2011. Aridification du climat régional et remontée de la limite inférieure du cèdre de l'Atlas (Cedrus atlantica Manetti) aux confins de la plaine de Midelt (Maroc). Physio-Géo. Géographie Physique et Environnement, 5 (1) : 143-165. https:// doi.org/10.4000/physio-geo.1983

Swets J. A., 1988. Measuring the accuracy of diagnostic systems. Science, New Series, 240 (4857): 1285-1293. https://doi.org/10.1126/science.3287615

Thomas C. D., Cameron A., Green R. E., Bakkenes M., Beaumont L. J., Collingham Y. C., et al., 2004. Extinction risk from climate change. Nature, 427 (6970): 145-148. https://doi.org/10.1038/nature02121
Thuiller W., Albert C., Araújo M. B., Berry P. M., Cabeza M., Guisan A., et al., 2008. Predicting global change impacts on plant species' distributions: Future challenges. Perspectives in Plant Ecology, Evolution and Systematics, 9 (3-4): 137-152. https://doi.org/10.1016/j.ppees.2007.09.004

Walther G.-R., Post E., Convey P., Menzel A., Parmesan C., Beebee T. J. C., et al., 2002. Ecological responses to recent climate change. Nature, 416 (6879): 389-395. https://doi.org/10.1038/416389a

Wisz M. S., Hijmans R. J., Li J., Peterson A. T., Graham C. H., Guisan A., 2008. NCEAS Predicting Species Distributions Working Group - Effects of sample size on the performance of species distribution models. Diversity and Distributions, 14 (5): 763-773. https://doi.org/10.1111/i.1472-4642.2008.00482.x

\section{Moukrim et al. - Contribution des auteurs}

\section{RÔLE DU CONTRIBUTEUR NOMS DES AUTEURS}

Conceptualisation

S. Moukrim, S. Lahssini

Gestion des données

S. Moukrim, S. Lahssini, H. Mharzi-Alaoui, K. Menzou, N. Rifai, A. Labbaci, I. Wahby, M. El Madihi

Analyse formelle S. Moukrim, S. Lahssini

Enquête et investigation S. Moukrim, H. MharziAlaoui, K. Menzou, N. Rifai, A. Labbaci, I. Wahby, M. El Madihi

\begin{tabular}{|c|c|}
\hline Méthodologie & S. Moukrim, S. Lahssini \\
\hline Gestion de projet & S. Moukrim \\
\hline Ressources & S. Moukrim, S. Lahssini \\
\hline Logiciels & S. Moukrim, S. Lahssini \\
\hline Supervision & $\begin{array}{l}\text { S. Lahssini, L. Rhazi, } \\
\text { M. Rhazi }\end{array}$ \\
\hline Validation & $\begin{array}{l}\text { S. Lahssini, L. Rhazi, } \\
\text { M. Rhazi }\end{array}$ \\
\hline Visualisation & S. Moukrim \\
\hline $\begin{array}{l}\text { Écriture - Préparation } \\
\text { de l'ébauche originale }\end{array}$ & S. Moukrim \\
\hline $\begin{array}{l}\text { Écriture - Révision } \\
\text { et édition }\end{array}$ & $\begin{array}{l}\text { S. Moukrim, S. Lahssini, } \\
\text { L. Rhazi, M. Rhazi, H. Mharzi } \\
\text { Alaoui, K. Menzou, N. Rifai, } \\
\text { A. Labbaci, I. Wahby, } \\
\text { M. El Madihi }\end{array}$ \\
\hline
\end{tabular}

Bois et Forêts des Tropiques - Revue scientifique du Cirad
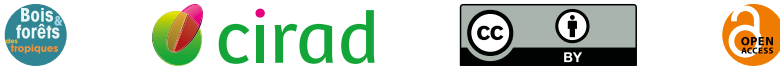

Cirad - Campus international de Baillarguet, 34398 Montpellier Cedex 5, France - Contact : bft@cirad.fr - ISSN : L-0006-579X 